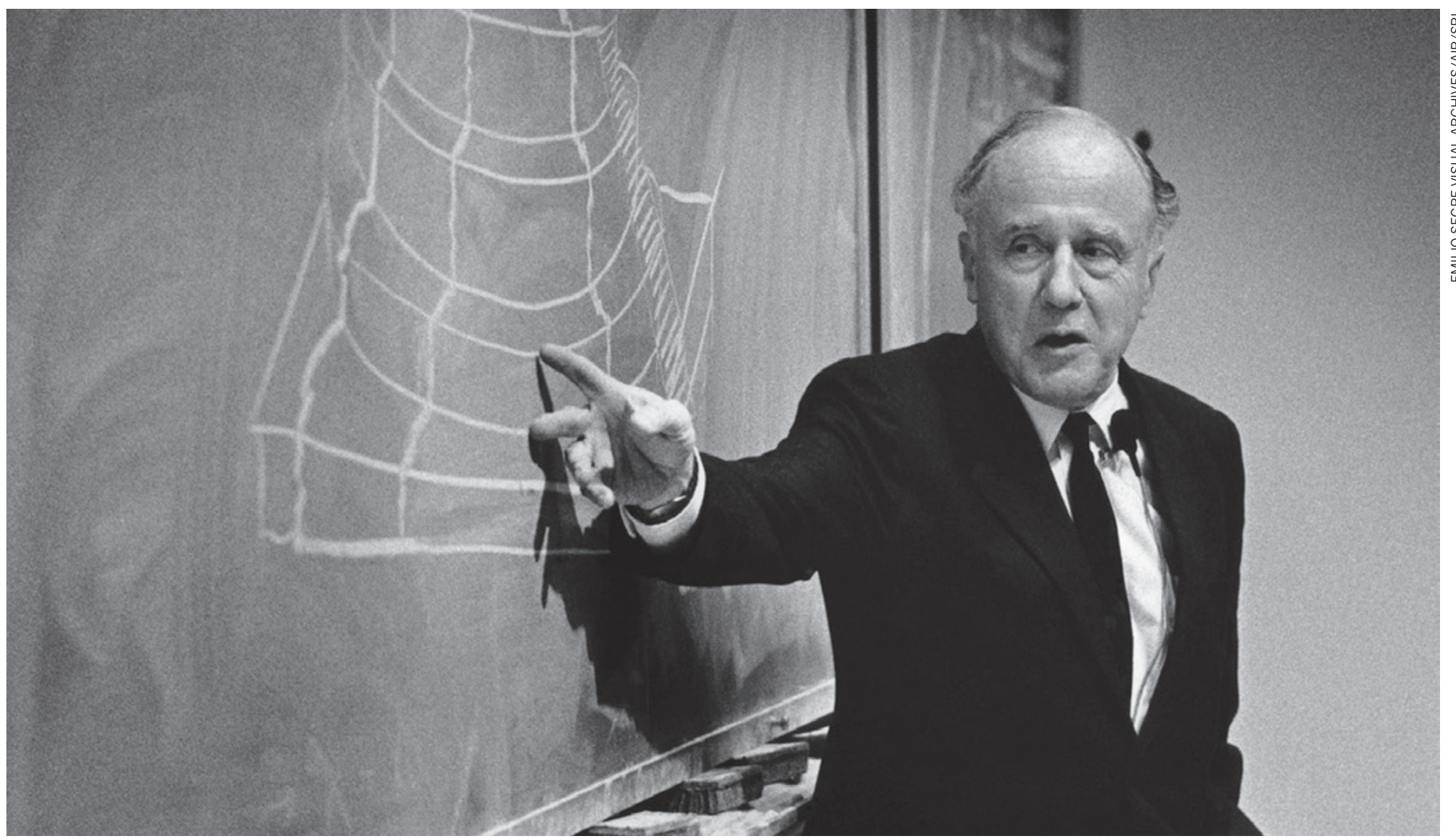

US theoretical physicist John Wheeler helped to bring general relativity into the mainstream.

\title{
Einstein's curve ball
}

\section{Graham Farmelo enjoys a 'biography’ of the general theory of relativity.}

$\mathrm{T}$ he mathematical physicist Max Born remarked in 1955 that although his late friend Albert Einstein's general theory of relativity was a peerless scientific achievement, "its connections with experience [are] slender". The appeal of the theory for Born was similar to that of "a great work of art, to be enjoyed and admired at a distance".

Today, Born's comments seem quaint. In an age of precision astronomy, it is now possible to study consequences of the theory; the existence of gravitational waves, for instance, can be inferred from studying pulsars. With the theory's centenary only a year away, this is an opportune time to look back on its inception and its achievements, as astrophysicist Pedro Ferreira does in The Perfect Theory, a 'biography' of Einstein's brainchild for those with a smattering of science and next to no mathematics.

Einstein recalled that his crucial epiphany occurred in 1907. Sitting in the Swiss patent office in Bern, he realized that "if a person falls freely he will not feel his own weight". Using what Born described as "the most amazing combination of philosophical penetration, physical intuition and mathematical skill", Einstein developed his general theory of relativity a new theory of gravity - and published it eight years later. In the final straight, the German mathematician David Hilbert was hot on his heels.

Ferreira outlines the theory, but I wish that he had tried a little harder to con-

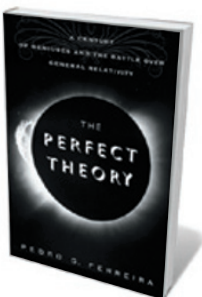

The Perfect Theory: A Century of Geniuses and the Battle over General Relativity PEDRO G. FERREIRA Houghton Mifflin Harcourt: 2014 vey the surpassing beauty of Einstein's equations. As the great theoretician Steven Weinberg has stressed, this was the quality that persuaded his colleagues to take relativity seriously. I suspect that many readers would have tolerated a few moments' perplexity for a sense of its mathematical glory.

The theory was past its fiftieth birthday when it entered mainstream physics. As
Ferreira describes, one of the most eminent of the physicists who brought the general theory back into the limelight was US theoretician John Wheeler. Wheeler was at first deeply uneasy about the theory's mathematical singularities - the point at which the quantities used to measure the strength of gravitational fields become infinite - and even wanted to remove them. In December 1963, he was one of the speakers at the first Texas Symposium on Relativistic Astrophysics, where the audience excitedly discussed the recently identified "quasi stellar radio sources", neatly dubbed quasars by one of the attendees. It seemed likely that general relativity might well be needed to understand this and other astronomical discoveries. Sure enough, the theory became a much more popular subject of study soon after, and several extremely strong research groups notably in Moscow; Princeton, New Jersey; and Cambridge, UK - began to catch the eye of the physics community.

At the symposium was the British mathematical physicist Roger Penrose, who went on to work with Stephen Hawking to make 
pioneering contributions to our understanding of the origins of the Universe, and of black holes, a term adopted by Wheeler after an audience member suggested it. Later, astronomers observed these exotic objects - regions of spacetime where gravity is so strong that it appears nothing can escape. Einstein would surely have been delighted to see this and other demonstrations of the surprising consequences of his theory. His equations were smarter than he was, to paraphrase the physicist Paul Dirac.

Yet general relativity is not quite perfect. It takes no account of quantum theory and is extremely difficult to

"General

relativity is now

the framework

for planning

and interpreting

many

astronomical

experiments."

combine with

the well-tested account of nature's other fundamental interactions weak, electromagnetic and strong - in the standard model

of particle physics. Ferreira lucidly sketches several attempts to generalize Einstein's theory, including string theory, which both describes gravity and offers an explanation of why it exists. Although enormously promising and mathematically rich, string theory is unpopular among some physicists in part because of the extreme difficulty of putting it to test, at least in the foreseeable future. Meanwhile, good old general relativity - once regarded as too recondite to be worth studying - is now the framework for planning and interpreting many astronomical experiments, as Ferreira describes in a moving coda.

When the sculptor Henry Moore visited Chicago, Illinois, in the late 1960s, the brilliant theoretical astrophysicist Subrahmanyan Chandrasekhar asked him how best to view a work of sculpture. Moore replied that the greatest of these works should be viewed from all distances, as new aspects of their beauty are revealed on every scale. Likewise, 50 years later, the mathematical aesthetic of relativity has been enhanced by the beautiful demonstrations of its veracity that Ferreira describes. These would probably have made Born ponder why he and his peers did not spend more time developing a deeper appreciation of the theory soon after Einstein first presented it. Maybe there's a lesson here for some of today's string-theory sceptics?

Graham Farmelo is a by-fellow at Churchill College, Cambridge, and author of Churchill's Bomb. e-mail:graham@grahamfarmelo.com

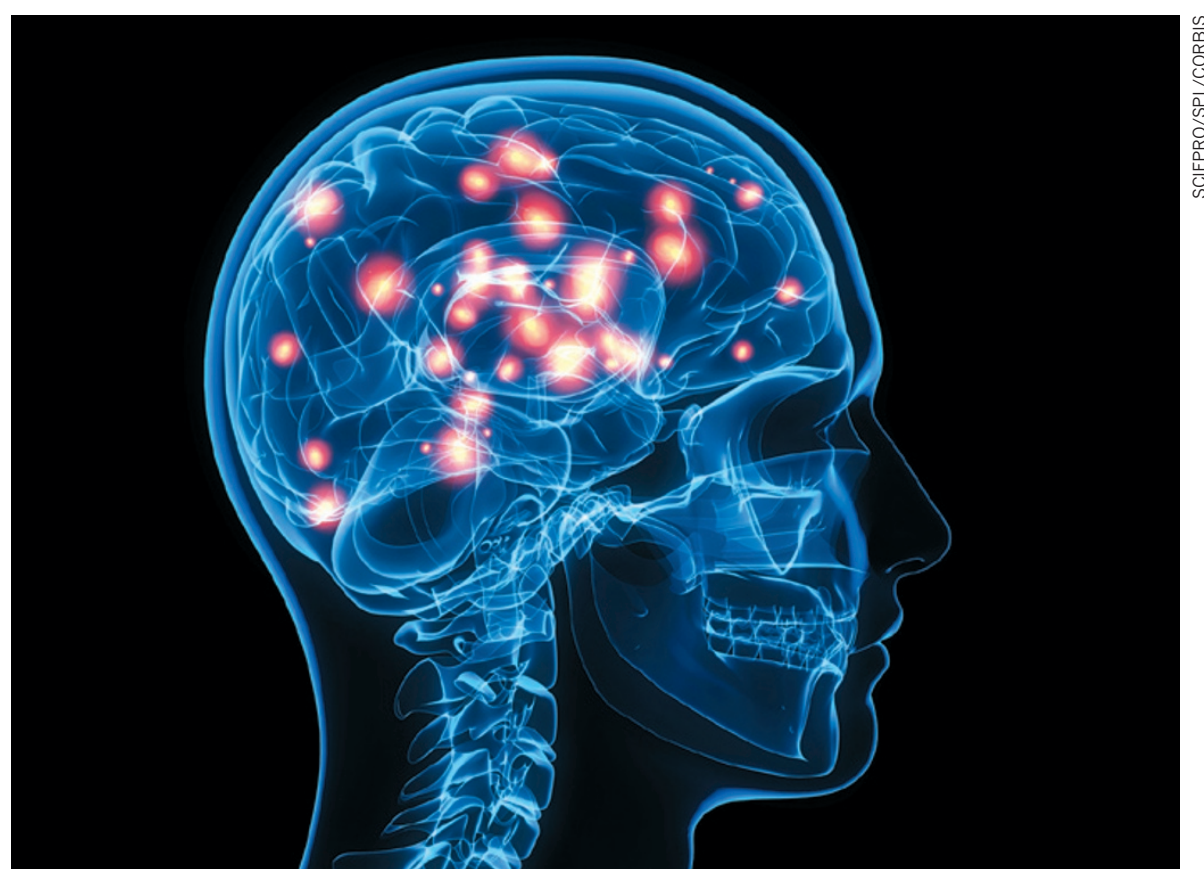

NEUROSCIENCE

\section{Joined-up thinking}

\section{Chris Frith explores a masterful model of how consciousness plays out in the theatre of the brain.}

$\lceil$ p n 1874, Thomas Henry Huxley gave a prescient lecture on mind and brain. The biologist argued that subjective experience depends on the brain's "anterior divisions", and that consciousness has as little effect on behaviour as a steam whistle has on a locomotive's progress - rendering humans little more than "conscious automata". He raised two questions that remain key in contemporary studies of the neural basis of consciousness: what is special about the neural processes that underlie consciousness, and what, if anything, is consciousness for?

The 1870 s seemed a likely time for a concerted research effort to answer those questions. Herman von Helmholtz had made the distinction between conscious and unconscious brain processes, and Gustav Theodor Fechner's 'psychophysics' had begun to allow the experimental study of the relationship between subjective experience and physical stimulation. But it was not until the 1970s that three-dimensional imaging of the living human brain became possible through physicist Peter Mansfield's work in magnetic resonance imaging. Among the first to realize the importance of this breakthrough for the study of mind and brain was cognitive neuroscientist Stanislas Dehaene. In his brilliant Consciousness and the Brain, Dehaene conveys the excitement of developing paradigms
Consciousness and the Brain:

Deciphering How the Brain Codes Our Thoughts STANISLAS DEHAENE Viking Books: 2014

on. He details many experiments, and presents the best attempt yet to answer the two questions raised by Huxley.

Regarding the first, on neural processes, the brain does a lot of work before we become conscious of a stimulus, as Helmholtz pointed out. When you read these words, you are rarely aware of the individual letters - yet you must have analysed them to have understood the meaning. How much unconscious analysis happens before what we are looking at emerges into consciousness? Dehaene relates how clever techniques have been developed to answer this question.

In backward masking, for example, a word (such as 'five') is presented, followed by a mask (a meaningless series of letters, for example). It has been found that the brain begins to analyse the word as soon as it appears, but that this analysis ceases when the mask appears. If the switch from word to mask is very rapid, there is no consciousness that the word was presented. Yet, as Dehaene has shown, the unconscious neural processing that goes 